\title{
Head-to-Head Comparison between High-Resolution Microultrasound Imaging and Multiparametric MRI in Detecting and Local Staging of Bladder Cancer: The BUS-MISS Protocol
}

Pietro Diana ${ }^{\mathrm{a}, \mathrm{b}, *}$, Giovanni Lughezzani ${ }^{\mathrm{a}, \mathrm{b}}$, Alberto Saita $^{\mathrm{b}}$, Alessandro Uleri ${ }^{\mathrm{a}, \mathrm{b}}$, Nicola Frego $^{\mathrm{a}, \mathrm{b}}$, Roberto Contieri $^{\mathrm{a}, \mathrm{b}}$, Nicolomaria Buffi ${ }^{\mathrm{a}, \mathrm{b}}$, Luca Balzarinic ${ }^{\mathrm{c}}$, Federico D’Orazio ${ }^{\mathrm{c}}$, Colombo Piergiuseppe $^{\mathrm{d}}$, Maria Grazia Elefante ${ }^{\mathrm{d}}$, Massimo Lazzeri ${ }^{\mathrm{b}}$, Giorgio Guazzoni ${ }^{\mathrm{a}, \mathrm{b}}$, Paolo Casale ${ }^{\mathrm{b}}$ and Rodolfo Hurle ${ }^{\mathrm{b}}$

${ }^{a}$ Department of Biomedical Sciences, Humanitas University, Milan, Italy

${ }^{\mathrm{b}}$ Department of Urology, IRCCS Humanitas Research Hospital, Milan, Italy

${ }^{\mathrm{c}}$ Department of Radiology, IRCCS Humanitas Research Hospital, Milan, Italy

${ }^{\mathrm{d}}$ Department of Pathology, IRCCS Humanitas Research Hospital, Milan, Italy

Received 27 September 2021

Accepted 12 January 2022

Pre-press 25 January 2022

Published 3 June 2022

\begin{abstract}
.
BACKGROUND: MRI has been proposed as a new staging tool for bladder cancer (BC), but use is limited by its high costs and low availability. 29-MHz high-resolution micro-ultrasound (mUS) technology has been suggested as an alternative to detect $\mathrm{BC}$ and distinguish between muscle-invasive and non-muscle invasive $\mathrm{BC}$.

OBJECTIVE: The aim was to compare the diagnostic accuracy of mUS vs. magnetic resonance imaging (MRI) in differentiating NMIBC and MIBC at definitive pathological examination.

METHODS: This is a prospective study of patients with a primary diagnosis of $\mathrm{BC}$ with either positive urine cytology (UC) or negative UC and a tumor size $>25 \mathrm{~mm}$ from a tertiary care high volume center. mUS, with the ExactVu system with an EV29L $29 \mathrm{MHz}$ side-fire transducer, and a 3-Tesla MRI were performed before transurethral resection of bladder tumor (TURBT) in every patient before undergoing TURBT. We compared the imaging results with pathological reports.

RESULTS: The analyzed population consisted of 58 individuals. The reported mUS and MRI sensitivity, specificity, positive, and negative predictive values were $85.0 \%, 76.3 \%, 65.4 \%$, and $90.6 \%$, versus $85.0 \%, 50.0 \%, 47.2 \%$, and $86.4 \%$, respectively. In accuracy analysis, the AUC for mUS and MRI were respectively 0.807 and 0.675 .
\end{abstract}

${ }^{*}$ Correspondence to: Pietro Diana, Via Baruffo 1, 26010 Ripalta Cremasca (CR), Italy. Tel.: +39 3927104765; E-mail: pietros. diana@gmail.com. 
CONCLUSIONS: In our population mUS seems to have a better performance in distinguishing NMIBC from MIBC. The main limitation of mUS is the probe shape that makes its use problematic in cases with a large prostate and inadequate rectal preparation. Further studies with a larger population are ongoing to compare and validate these techniques in this setting.

Keywords: Bladder cancer, diagnosis, microultrasound, magnetic resonance, pre-operative local staging, technology, treatment strategy

\section{INTRODUCTION}

Bladder cancer (BC) can be differentiated between non-muscle-invasive BC (NMIBC) and muscleinvasive $\mathrm{BC}$ (MIBC), with the latter being associated with a poorer prognosis. Cancer staging is crucial in determining the best therapeutic strategy $[1,2]$. The gold standard for assessing BC local stage and grade is transurethral resection (TUR), which can be used both for local staging and treatment in cases of localized cancer [3, 4]. However, this approach is not devoid of risks: it is an invasive endoscopic procedure requiring anesthesia with inherent surgical hazards, especially in patients with hemorrhagic diathesis and those undergoing anticoagulant therapy. Thus, a non-invasive diagnostic and staging technique is warranted in this kind of pathology.

Multiparametric magnetic resonance imaging (MRI) is gaining a role in the differentiation between NMIBC and MIBC [5, 6]. The use of a MRI-based standardized reporting system, the Vesical Imaging Reporting and Data System (VI-RADS) [7] has been suggested to further improve the characterization of the tumor and peer-to-peer communication $[6,8,9]$. MRI contraindications, cost, and availability is limiting the diffusion of this technique [10].

In the recent years, the $29-\mathrm{MHz}$ high-resolution microultrasound (mUS) technology has been suggested as an effective tool in the diagnosis of clinically-significant prostate cancer (csPCa) [1113]. The benefit of this technology is the extremely high resolution, down to 70 microns, which provides a significant improvement in the visualization of tissue details compared with standard US. In the setting of $\mathrm{BC}$, preliminary findings showed that mUS is able to differentiate the healthy bladder wall layers, identify and differentiate bladder cancer between NMIBC form MIBC [14]. mUS may thus provide a real-time evaluation and cost effectiveness strategy $[14,15]$.

The aim of this prospective comparative study is to evaluate and confirm the potential role of mUS in the identification and characterization of $\mathrm{BC}$ in in a head-to-head comparison to MRI through a histopathological confirmation of the results.

\section{MATERIALS AND METHODS}

This is a prospective comparative single-center study including both male and female patients older than 18 year-old referred to our tertiary urological center with a primary diagnosis of $\mathrm{BC}$ by ultrasound and flexible cystoscopy. The diagnosis was required to be made in our institution in order to have the complete pre-operative data and images.

Inclusion criteria were: 1) patients presenting with a primary diagnosis of $\mathrm{BC} ; 2$ ) the lesion had to be larger than $25 \mathrm{~mm}$ or had to present a positive urine cytology. Exclusion criteria included: 1) patients presenting with more than 3 lesions at diagnosis (as the objective is to clearly characterize each lesions);2) positive urine culture 3 ) history of acute or chronic urinary retention or vesical catheter holder; 3 ) patients subjected to previous endoscopic radiotherapy and/or chemotherapy treatment; 4) patients with urethral, anal, and/or vaginal stricture; 5) patients with hip problems that do not allow the lithotomic position; 7) patients with a BMI > 35. Diagnosis was made on a base of either flexible cystoscopy, transabdominal ultrasonography, or computed tomography (CT). Patients were subsequently scheduled for an endoscopic treatment.

MRI was performed using a 3 Tesla magnet, equipped with a 32-channel phased-array coil. The imaging protocol of the pelvis, focused on the bladder, was in line for VI-RADS-assessment MRI images reading was performed by two radiologists (L.B. and F.D.), both with an experience of more than 5 years.

Prior to the TUR, all patients underwent highresolution mUS assessment in a lithotomic position. Micro-US imaging was performed using the ExactVu system with an EV29L 29-MHz side- fire transducer (Exact Imaging, Markham, Canada). Two urologists (G.L. and M.L.), who were routinely using mUS for the detection of $\mathrm{PCa}$, and who were blinded to the number and location of the lesions, performed the assessment. The bladder was previously emptied by catheterization and afterward filled with $50-100 \mathrm{cc}$ of sterile saline solution. The mUS was performed 
transrectally in males and transvaginally in females, and a rectal enema was performed in the male population before the procedure. Images and recording of the bladder were analyzed according to the previously mentioned study [14].

The surgical procedure was performed according to the standard technique at our institution which are in line with the EAU guidelines and take into account the TURBT pre-operative checklist $[16,17]$.

Primary endpoint were to perform a head-to-head comparison of mUS vs. MRI in identifying and accurately characterizing $\mathrm{BC}$ in terms of number and stage of the lesions. Secondly, we assessed the accuracy of both mUS and MRI to discriminate NMIBC from MIBC comparing the mUS findings with the histopathological results. All image sets were analyzed in a double blind fashion and before the histopathological analysis. Patients received a either an En-Bloc TUR or a standard bipolar TUR depending on the number and dimension of the lesions [18].

All specimens were fixed in $10 \%$ formalin, embedded in paraffin, cut, and stained with hematoxylin and eosin. Specimens were examined by two expert uropathologists to assess the type, grade, and stage of the tumor. Malignant tumors were classified and graded according to the World Health Organization classification [19]. Tumor staging was defined according to the American Joint Committee on Cancer/Union for International Cancer Control TNM system [20].

\section{Ethics}

The institutional review board and local ethical committee approved the study (Protocol number 1322 code ICH-008). A written informed consent has been signed by all patients that were included in this study.

\section{Statistical analysis}

In order to estimate the concordance between the two methods, we want to exclude a concordance inferior to $80 \%$, so the lower limit of the confidence interval will not include 0.8 . Thus, at least 50 patients should be included into the study.

Demographic and perioperative data were analyzed using descriptive statistics. Association with concordance will be explored with logistic regression analysis, reporting OR with $95 \%$ confidence interval. A $p$-value $<0.05$ will be considered statistically significant. Predictive accuracy values were quantified as the ROC AUC with a value of $100 \%$ indicating perfect prediction and $50 \%$ equivalent to the toss of a coin. All analyses were performed using IBM SPSS version 23 (SPSS Inc., Chicago, IL, USA).

\section{RESULTS}

Between April 2018 and November 2020, we prospectively screened 72 patients with primitive $\mathrm{BC}$. Five patients $(6.9 \%)$ were excluded because could not undergo MRI for either chronic kidney disease or presence of non-compatible metallic implants. One patient $(1.4 \%$ ) was subjected to TUR in an emergency setting in another hospital for anemia, one patient (1.4\%) was excluded because unfit for an endoscopic procedure for cardiac comorbidities, and one (1.4\%) because of metastatic disease at preoperative staging. The mUS procedure was feasible in all female patients, while we failed to evaluate 4 male patients $(5.5 \%)$ due to the longitudinal length of the prostate $(>6 \mathrm{~cm})$ resulting in a suboptimal bladder window. Two patients $(2.8 \%)$ resulted negative for neoplasia at histopathological analysis even though a tumor looking mass was diagnosed with both cystoscopy and abdominal US and then confirmed both by MRI and mUS as such. In one case, histology reported a mixed B- and T-cell inflammatory non-neoplastic solid aggregate and in the other case chronic cystitis was found in a context of schistosomiasis. The algorithm is depicted in Fig. 1.

Final analysis was performed on 58 patients. Demographics, clinical features, and pre-operative data are summarized in Table 1. Intra-operative data and pathologic description are described in Table 2. Fourty-four $(75.9 \%)$ patients underwent traditional TUR, fourteen $(24.1 \%)$ patients were treated with En-Bloc resection. Detrusor muscle (DM) was not retrieved in the pathological sample in 2 patients (3.4\%) that resulted Ta HG tumors, but at reTURBT residual tumor was found in one patient (pTaLG). mUS and MRI imaging is depicted in Figs. 2, 3, and 5 .

When comparing mUS and final pathology findings (Table 3), 29/32 (90.6\%) NMIBC cases and 17/26 (65.4\%) MIBC cases were correctly staged by mUS; conversely, 3/32 (9.4\%) NMIBC cases were upstaged to MIBC and 9/26 (34.6\%) MIBC cases were downstaged to NMIBC at histopathological evaluation. The reported sensitivity, specificity, and positive, and negative predictive values for mUS 


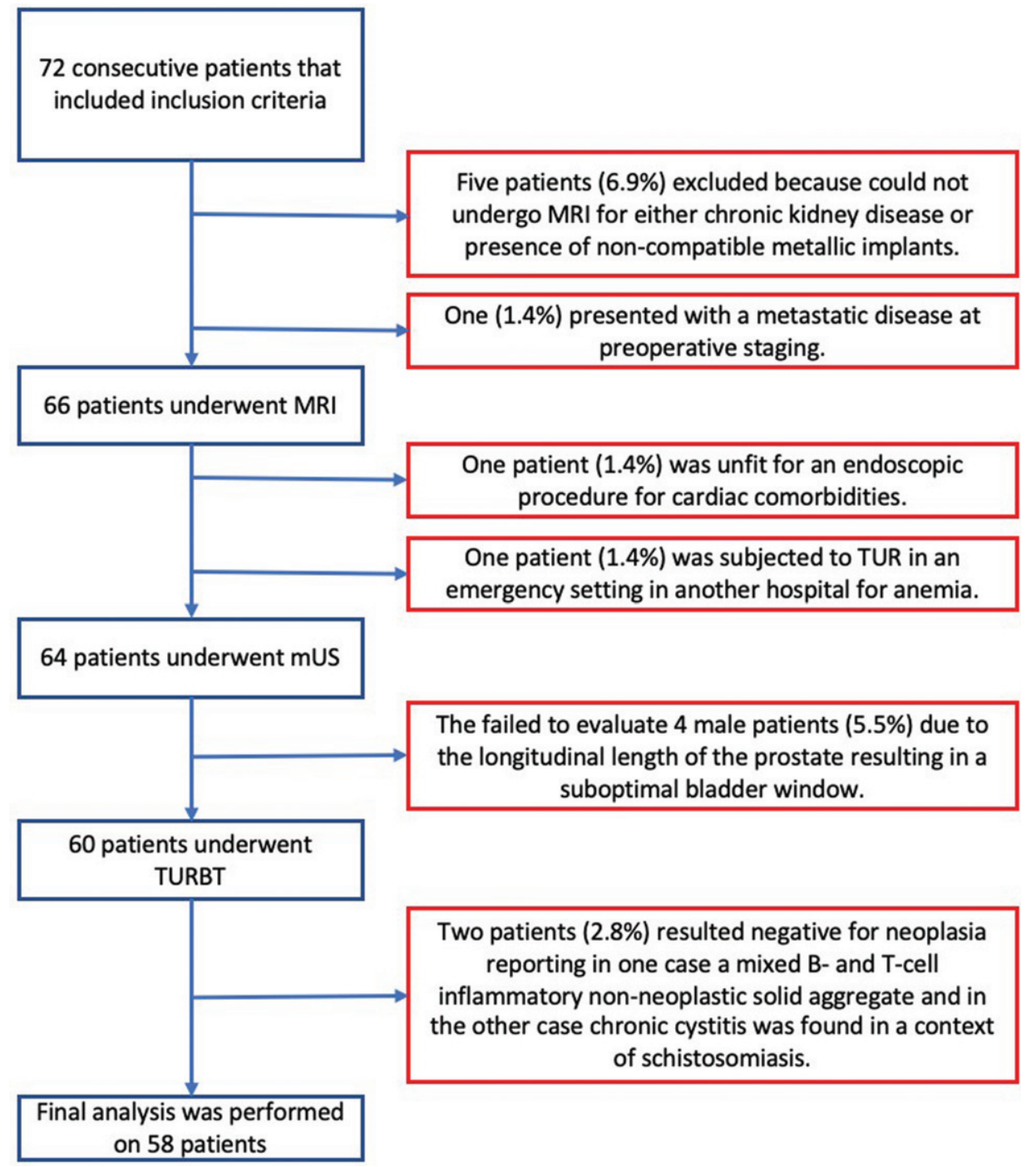

Fig. 1. Patients inclusion and exclusion algorithm (abbreviations: mUS: microultrasound; MRI: magnetic resonance imaging; TURBT: transurethral resection of bladder tumor).

were $85.0 \%, 76.3 \%, 65.4 \%$, and $90.6 \%$, respectively. When evaluating MRI staging (Table 3), the pathologists confirmed 19/22 (86.4\%) cases of NMIBC and $17 / 36$ cases $(47.2 \%)$ of MIBC; instead, 3 case of $22(13.6 \%)$ NMIBC cases was upstaged to MIBC and 19/36 (52.8\%) MIBC cases were downstaged to NMIBC at histopathological evaluation. The reported sensitivity, specificity, and positive, and negative predictive values for MRI were $85.0 \%, 50.0 \%, 47.2 \%$, and $86.4 \%$, respectively. Figure 4 depicts the ROC curve for the staging accuracy of both mUS and MRI. The AUC for mUS and MRI reach 0.807 and 0.675 , respectively in discriminating MIBC from NMIBC.

\section{DISCUSSION}

Our findings show that mUS is feasible and effective in the detection and local staging of primary BC high risk patients. The concordance between mUS and histopathological results seems to confirm that mUS could be used as a noninvasive, and potentially cost-effective staging device in primary BC.

Conventional Bladder US in the diagnosis of BC has been examined and showed to have a sensibility of $72-87 \%$ for the detection of tumors [21,22]. Other modalities such as contrast-enhanced US (CEUS) have been investigated to increase the detection and the accuracy other tumor features such as tumor grade 
Table 1

Demographic and Pre-operative data(abbreviations: mUS: microultrasound; MRI: magnetic resonance imaging; NMIBC: non muscle invasive bladder cancer; MIBC: muscle invasive bladder cancer)

\begin{tabular}{ll}
\hline \multicolumn{2}{c}{ Demographic Data } \\
\hline Gender, $n(\%)$ & $-47(81.0 \%)$ \\
- Male & $-11(19.0 \%)$ \\
- Female & $-71.5(12.75)$ \\
Age, median (IQR) & \\
Smoker, $n(\%)$ & $-14(24.2 \%)$ \\
- No & $-22(37.9 \%)$ \\
- Yes & $-22(37.9 \%)$ \\
- Former & \\
Macrohematuria, $n(\%)$ & $-5(8.6 \%)$ \\
- No & $-53(91.4 \%)$ \\
- Yes & $-28(48.3 \%)$ \\
Urinary cytology, $n(\%)$ & $-18(31.0 \%)$ \\
- Negative & $-12(20.7 \%)$ \\
- Positive & 1.34 \\
- Atypia & $30(15)$ \\
Number of lesion/patient & \\
Principallesiondimension, median (IQR) & \\
\hline & MRI \\
\hline Lesion stage, $n(\%)$ & $-22(37.9 \%)$ \\
- NMIBC & $-36(62.1 \%)$ \\
- MIBC & $-1(1.7 \%)$ \\
VIRADS, n $(\%)$ & $-20(34.5 \%)$ \\
- 1 & $-5(8.6 \%)$ \\
- 2 & $-15(25.9 \%)$ \\
- 3 & $-17(29.3 \%)$ \\
- 4 & \\
- 5 & $-26(44.8 \%)$ \\
\hline & \\
\hline Lesion stage, $n(\%)$ & \\
- NMIBC & \\
- MIBC & \\
\hline
\end{tabular}

and depth of invasion [21]. Nicolau et al have compared CEUS with US and showed an improvement in tumor detection with a rate of $83 \%$ vs $72 \%$ and ability to detect more tumors per patient with a rate of $66 \%$ vs $61 \%$. Both modalities were poor in detecting small lesions $29 \%<5 \mathrm{~mm}$ versus $95 \%$ for lesions $>5 \mathrm{~mm}$ [21]. CEUS with echocolordoppler technology improves sensibility from $87 \%$ to $92 \%$ specificity from $60 \%$ to $85.5 \%$ [23, 24]. No success was achieved in the description of other tumor characteristics.

MRI including functional sequences has been suggested to be able to discriminate between NMIBC and MIBC [6, 8, 25-27]. This imaging technique could potentially result in overstaging as tumor-associated fibrosis or inflammation can mimic the low signal intensity of the muscularis propria [28]. To overcome these limitations, the VI-RADS score has been developed to help defining and standardizing the grade of $\mathrm{BC}$ invasion $[6,8,27]$. However, potential drawbacks
Table 2

Peri-operative and Pathologic data(abbreviations: IQR: inter quartile range; LG: low grade; HG: high grade; NMIBC: non muscle invasive bladder cancer; MIBC: muscle invasive bladder cancer)

\begin{tabular}{|c|c|}
\hline \multicolumn{2}{|c|}{ Operative and Pathologic data } \\
\hline Number of lesion/patient, median (IQR) & 1.35 \\
\hline Principallesiondimension, median (IQR) & $30(20)$ \\
\hline \multicolumn{2}{|l|}{ Lesion site, $n(\%)$} \\
\hline - Trigone & $-6(10.3 \%)$ \\
\hline - Left lateral wall & $-22(37.9 \%)$ \\
\hline - Right lateral wall & $-21(36.2 \%)$ \\
\hline - Posterior wall & $-4(6.9 \%)$ \\
\hline - Anterior wall & $-1(1.7 \%)$ \\
\hline - Doom & $-3(5.2 \%)$ \\
\hline - Multifocal & $-1(1.7 \%)$ \\
\hline \multicolumn{2}{|l|}{ Appearance } \\
\hline - Papillary & $-18(31.0 \%)$ \\
\hline - Sessile & $-37(63.8 \%)$ \\
\hline - Flat & $-3(5.2 \%)$ \\
\hline \multicolumn{2}{|l|}{ En-Bloc } \\
\hline- No & $-44(75.9 \%)$ \\
\hline - Yes & $-14(24.1 \%)$ \\
\hline \multicolumn{2}{|l|}{ Lesion pT } \\
\hline$-\mathrm{Ta}$ & $-27(46.5 \%)$ \\
\hline$-\mathrm{T} 1$ & $-11(19.0 \%)$ \\
\hline$-\mathrm{T} 2$ & $-20(34.5 \%)$ \\
\hline \multicolumn{2}{|l|}{ Lesion grade (WHO1973 classification) } \\
\hline$-\mathrm{G} 1$ & $-10(17.2 \%)$ \\
\hline$-G 2$ & $-13(22.4 \%)$ \\
\hline$-\mathrm{G} 3$ & $-35(60.3 \%)$ \\
\hline \multicolumn{2}{|l|}{ Lesion grade(WHO 2004 classification) } \\
\hline$-\mathrm{LG}$ & $-14(24.1 \%)$ \\
\hline$-\mathrm{HG}$ & $-44(75.9 \%)$ \\
\hline \multicolumn{2}{|l|}{ Lesion stage } \\
\hline - NMIBC & $-38(65.5 \%)$ \\
\hline - MIBC & $-20(34.5 \%)$ \\
\hline
\end{tabular}

MRI have to be considered, such as costs and causes of artifacts [29]. Recent studies also showed concordance between experienced and unexperienced readers [26], and a prospective analysis has also been published confirming the previous results [27].

Our cohort takes into account a subset of population that differs from the ones that have been analyzed in the previously published studies evaluating the staging accuracy of MRI. In the current prospective study we decided to adopt strict inclusion criteria on lesion dimension and/or positive urine cytology, resulting in a population having a higher risk of harboring MIBC, observed in $32.7 \%$ of patients, and high grade tumors, observed in $75.9 \%$ of patients.

In the literature, a relationship between VI-RADS and tumor dimension is intuitively and clearly present, as the great majority of patients with suspected MIBC at mpMRI (VI-RADS $\geq 3$ ) had a tumor size greater than $3 \mathrm{~cm}$, and a selection bias may therefore be operational [27]. Additionally, in cohort from Del Giudice et al the actual accuracy in detecting 


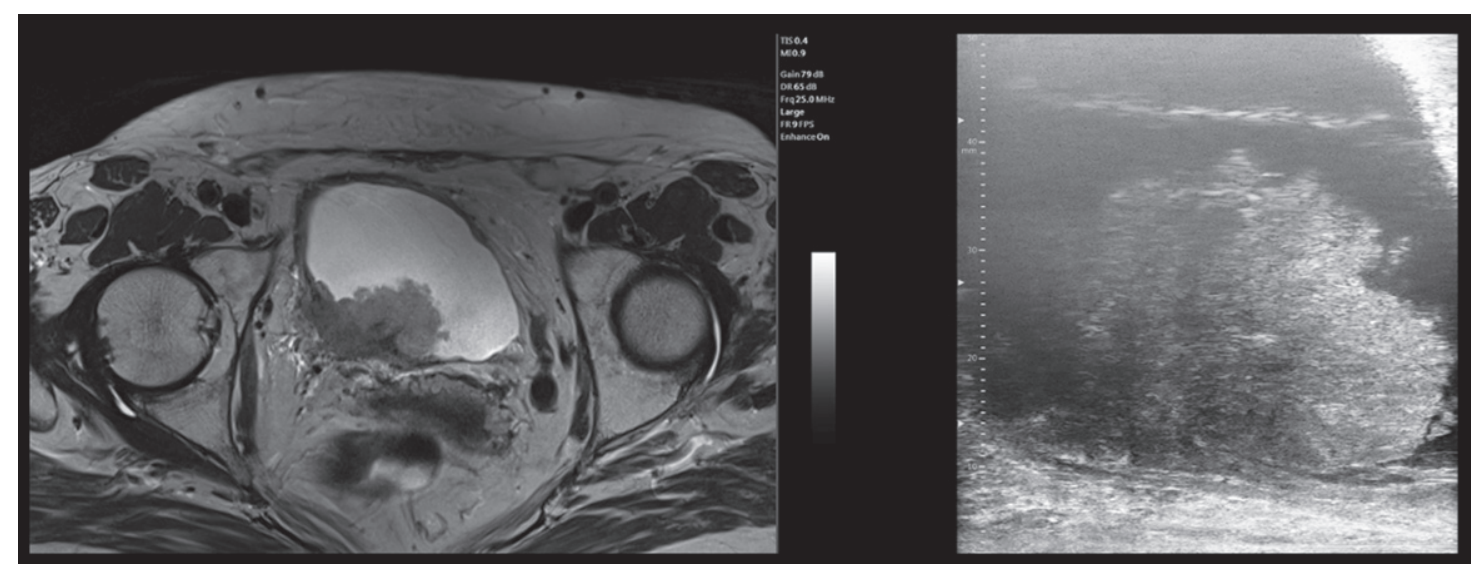

Fig. 2. mUS and MRI imaging comparison, patient 1. In the mUS the tumor disrupts the three layer structure showing a mixed hypo and hyperechoic pattern and at MRI the tumor extends beyond the bladder wall. In both techniques a single large stalk can be detected. (abbreviations: mUS: microultrasound; MRI: magnetic resonance imaging).

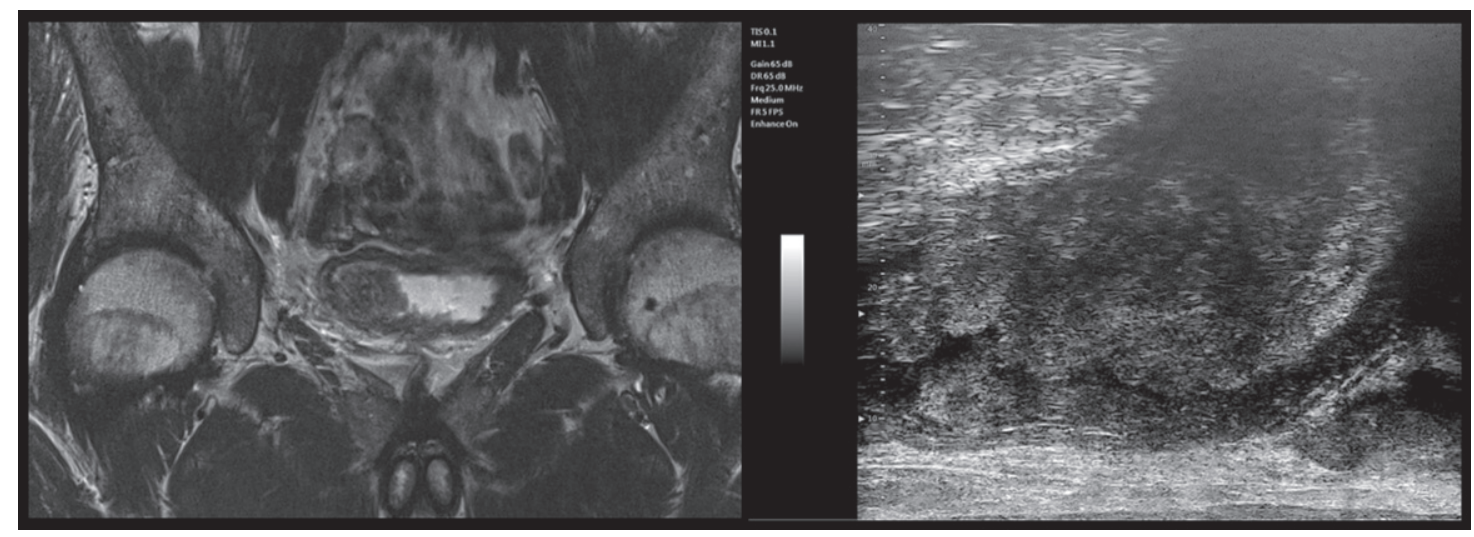

Fig. 3. mUS and MRI imaging comparison, patient 2. In both the mUS and mpMRI imaging the tumor is clearly extending into the muscular layer, showing a hyperechoic aspect at the base of the lesion associated with a "starry sky" pattern and a clear extension beyond the bladder wall in MRI. (abbreviations: mUS: microultrasound; MRI: magnetic resonance imaging).

Table 3

mUS and MRI Staging comparison with pathological staging(abbreviations: mUS: microultrasound; MRI: magnetic resonance imaging; NMIBC: non muscle invasive bladder cancer; MIBC: muscle invasive bladder cancer)

\begin{tabular}{|c|c|c|c|c|}
\hline Technique & Staging & $n$ & Histological vs Imaging Staging & $n(\%)$ \\
\hline \multirow[t]{2}{*}{ mUS } & NMIBC, $n$ & 32 & $\begin{array}{l}\text { - Correct staging } \\
\text { - Upstaging }\end{array}$ & $\begin{array}{l}\text { - } 29(90.6 \%) \\
\cdot 3(9.4 \%)\end{array}$ \\
\hline & MIBC, $n$ & 26 & $\begin{array}{l}\text { - Correct staging } \\
\text { - Downstaging }\end{array}$ & $\begin{array}{l}\text { - } 17(65.4 \%) \\
\cdot 9(34.6 \%)\end{array}$ \\
\hline \multirow[t]{2}{*}{ MRI } & NMIBC, $n$ & 22 & $\begin{array}{l}\text { - Correct staging } \\
\text { - Upstaging }\end{array}$ & $\begin{array}{l}\text { - } 19(86.4 \%) \\
\text { - } 3(13.6 \%)\end{array}$ \\
\hline & MIBC, $n$ & 36 & $\begin{array}{l}\text { - Correct staging } \\
\text { - Downstaging }\end{array}$ & $\begin{array}{l}\text { - } 17(47.2 \%) \\
\text { - } 19(52.8 \%)\end{array}$ \\
\hline
\end{tabular}

MIBC by looking at the VIRADS scores diagnostic for MIBC (VIRADS 4 and 5) there is a large number of patients that result NMIBC at the histopathological analysis. Thus, the enthusiasm in proposing this system as substitute of TURBT is impractical and the consequence on the quality of life of patients would be dramatic as about $40 \%$ of radical cystectomies performed on the mpMRI data would be unnecessary. 


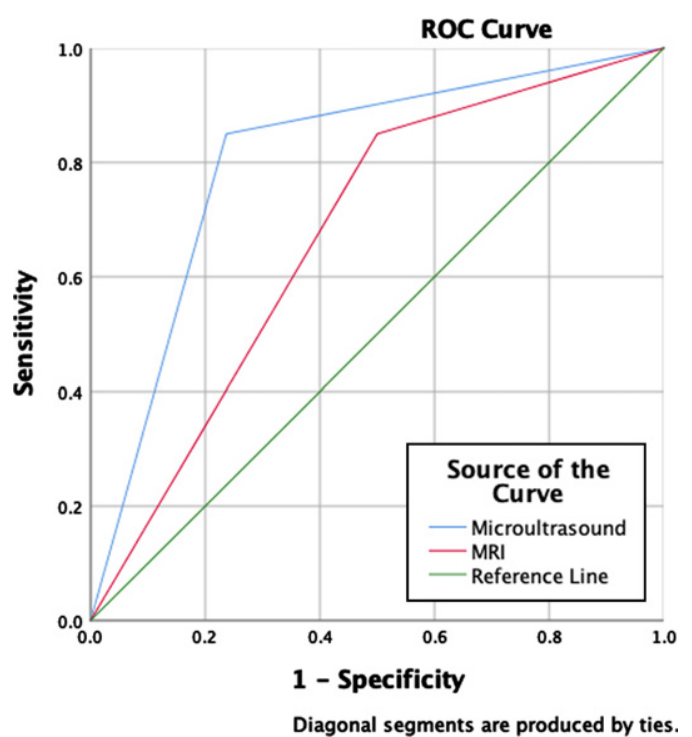

\begin{tabular}{|c|c|}
\hline Diagnostic tool & AUC \\
\hline MRI & 0.675 \\
\hline Microultrasound & 0.807 \\
\hline
\end{tabular}

Fig. 4. ROC Curve and AUCs for the staging accuracy of both mUS and MRI. (abbreviations: mUS: microultrasound; MRI: magnetic resonance imaging).
Consequently, it is not clear whether MRI should be reserved to the diagnostic work-up of patients at high risk for MIBC in order to discriminate those that would benefit from surgery, neoadjuvant chemotherapy, directly be treated with bladder-sparing therapies or trimodal therapy.

In our experience, we were able to detect the great majority of BC and we found a good correlation between mUS and histopathological results. mUS feasibility in the $\mathrm{BC}$ setting was already been reported by Saita et al. [14], who were able to describe the normal anatomy and preliminary results suggesting the potential role of mUS for tumor characterization were shown. Despite a correlation with the histopathological analysis was reported, the main limitation was the small population size.

The strength of this study is related to the completeness of pre- and intra-operative data of patients according to the TURBT checklist that assures a good quality procedure and a clear patient clinical picture. Another crucial point is the patient selection; We decided to include in the study only patients with primary bladder lesions in order to have a standard bladder wall and thus a standard mUS window. The nature of our study is prospective and it is the first
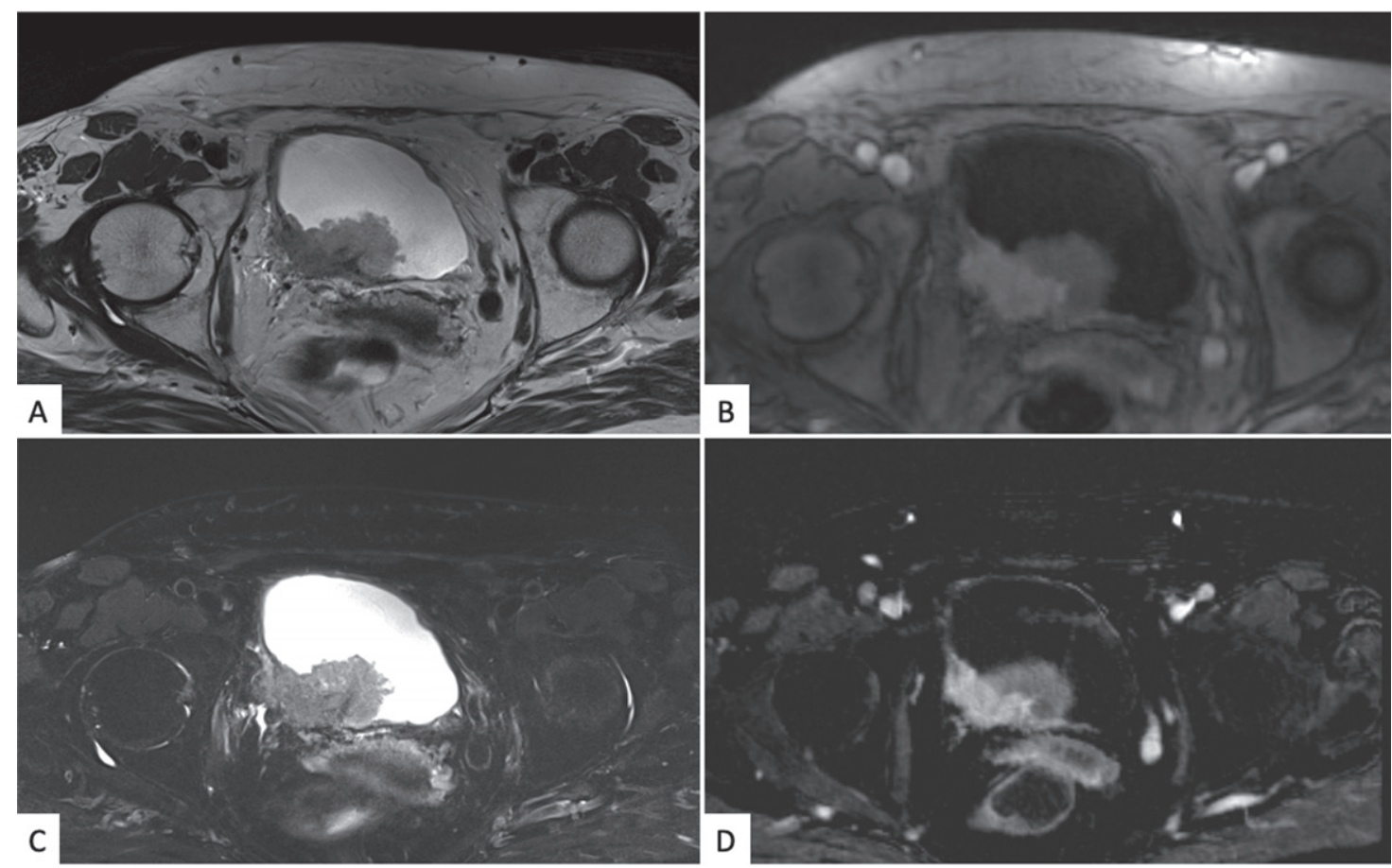

Fig. 5. Bladder mpMRI: axial projection T2 weighted (A), DWI and ADC imaging (B and C), and dynamic imaging in arterial phase after paramagnetic contrast medium injection (D), The images show the presence of a large heteroplastic lesion of the poster bladder wall invading the fatty tissue around the bladder. (abbreviations: MRI: magnetic resonance imaging; DWI: diffused weighted imaging). 
large sample of BC patients analyzed with the new technology of mUS and the first comparative study in this setting with both the mpMRI and the histopathological analysis.

Our study is not devoid of limitations; first, limitations linked to the procedure are the probe shape, which was not designed for bladder visualization still leading to a successful acquisition of an acceptable mUS window in most patients analyzed in our study $(94.5 \%)$; large prostates $(>6 \mathrm{~cm}$ in longitudinal length) and inadequate rectal preparations in men were the main obstacle for an adequate echographic visualization of the bladder. The mUS provides only longitudinal sections of the bladder, thus, its accuracy to determine the location of a lesion may be suboptimal with a dislocation of lesions located on the anterior wall and doom of the bladder, but with a very high accuracy in those located on the posterior wall or trigone. Both mUS and mpMRI do not provide a histopathological specimen. At last, there is no formal comparison of neither mUS or mpMRI with CT-urography as institutional protocol consider it only for patients affected by MIBC.

Applications for this staging tool are several. Comorbid and frail patients and those taking antiaggregant therapy for cardiovascular surgeries would undergo an endoscopic intervention with a higher risk of intra and post-operative complications and a noninvasive mUS evaluation could help discrimination those cases in which it would be safer to undergo or, on the contrary, delay the procedure, or avoid reTUR [15]. This technique can be further employed in active surveillance protocols and in the follow-up of patients undergoing neoadjuvant and trimodal therapy in order to gain easily new information about the disease persistence and progression.

Future perspectives in the use of the novel 29$\mathrm{MHz}$ high-resolution mUS are the development of a scoring system that will permit a higher precision and accuracy in the discrimination of NMIBC from MIBC. Similar score has already been developed in the PCa setting where Ghai et al. [30] developed the PCa risk identification system mUS (PRI-MUS), which could guide urologists to achieve accurate and reproducible results for $\mathrm{PCa}$ detection comparable with those reported by MRI-based PI-RADS score [31, 32].

\section{ACKNOWLEDGMENTS}

The authors have no acknowledgments.

\section{FUNDING}

The authors report no funding

\section{AUTHOR CONTRIBUTIONS}

Pietro Diana: conception, performance of work, writing the article

Giovanni Lughezzani: conception, performance of work, writing the article

Alberto Saita: performance of work

Alessandro Uleri: performance of work

Nicola Frego: performance of work

Roberto Contieri: performance of work

Nicolomaria Buffi: interpretation of data

Luca Balzarini: conception

Federico D'Orazio: conception

Colombo Piergiuseppe: interpretation of data

Maria Grazia Elefante: interpretation of data

Massimo Lazzeri: conception

Giorgio Guazzoni: conception

Paolo Casale: interpretation of data

Rodolfo Hurle: conception, writing the article

\section{CONFLICT OF INTEREST}

None of the authors (Pietro Diana, Giovanni Lughezzani, Alberto Saita, Alessandro Uleri, Nicola Frego, Roberto Contieri, Nicolomaria Buffi, Luca Balzarini, Federico D’Orazio, Colombo Piergiuseppe, Maria Grazia Elefante, Massimo Lazzeri, Giorgio Guazzoni, Paolo Casale, Rodolfo Hurle) have conflicts of interest to report.

\section{REFERENCES}

[1] Seiler R, Ashab HAD, Erho N, et al. Impact of Molecular Subtypes in Muscle-invasive Bladder Cancer on Predicting Response and Survival after Neoadjuvant Chemotherapy [Figure presented]. Eur Urol. 2017. doi:10.1016/j.eururo.2017.03.030

[2] D'Andrea D, Soria F, Zehetmayer S, et al. Diagnostic accuracy, clinical utility and influence on decision-making of a methylation urine biomarker test in the surveillance of non-muscle-invasive bladder cancer. BJU Int. 2019. doi:10.1111/bju.14673

[3] Munari E, Caliò A, Brunelli M, Martignoni G. Predicting progression in T1 non-muscle-invasive bladder cancer: back to histology. BJU Int. 2018. doi:10.1111/bju.14465

[4] Colombo R, Hurle R, Moschini M, et al. Feasibility and Clinical Roles of Different Substaging Systems at First and Second Transurethral Resection in Patients with T1 High-Grade Bladder Cancer. Eur Urol Focus. 2018. doi:10.1016/j.euf.2016.06.004 
[5] Lee M, Shin SJ, Oh YT, et al. Non-contrast magnetic resonance imaging for bladder cancer: fused high $b$ value diffusion-weighted imaging and T2-weighted imaging helps evaluate depth of invasion. Eur Radiol. 2017. doi:10.1007/s00330-017-4759-2

[6] Gandhi N, Krishna S, Booth CM, et al. Diagnostic accuracy of magnetic resonance imaging for tumour staging of bladder cancer: systematic review and meta-analysis. BJU Int. 2018. doi:10.1111/bju.14366

[7] Panebianco V, Barentsz J, Narumi Y, Catto J. Reply to Andrea Necchi, Antonella Messina, and Alberto Briganti's Letter to the Editor re: Valeria Panebianco, Yoshifumi Narumi, Ersan Altun, et al. Multiparametric Magnetic Resonance Imaging for Bladder Cancer: Development of VI-RADS (Vesical Imaging-Rep). Eur Urol. 2018. doi:10.1016/j.eururo.2018.06.029

[8] Panebianco V, Narumi Y, Altun E, et al. Multiparametric Magnetic Resonance Imaging for Bladder Cancer: Development of VI-RADS (Vesical Imaging-Reporting And Data System). Eur Urol. 2018. doi:10.1016/j.eururo.2018.04.029

[9] Woo S, Panebianco V, Narumi Y, et al. Diagnostic Performance of Vesical Imaging Reporting and Data System for the Prediction of Muscle-invasive Bladder Cancer: A Systematic Review and Meta-analysis. Eur Urol Oncol. 2020;0(0):1-10. doi:10.1016/j.euo.2020.02.007

[10] Thoeny HC, Forstner R, De Keyzer F. Genitourinary applications of diffusion-weighted MR imaging in the pelvis. Radiology. 2012. doi:10.1148/radiol.12110446

[11] Lughezzani G, Saita A, Lazzeri M, et al. Comparison of the Diagnostic Accuracy of Micro-ultrasound and Magnetic Resonance Imaging/Ultrasound Fusion Targeted Biopsies for the Diagnosis of Clinically Significant Prostate Cancer. Eur Urol Oncol. 2019;2(3):329-32. doi:10.1016/j.euo.2018. 10.001

[12] Lughezzani G, Maffei D, Saita A, et al. Diagnostic Accuracy of Microultrasound in Patients with a Suspicion of Prostate Cancer at Magnetic Resonance Imaging: A Singleinstitutional Prospective Study. Eur Urol Focus. 2020. doi:10.1016/j.euf.2020.09.013

[13] Abouassaly R, Klein EA, El-Shefai A, Stephenson A. Impact of using $29 \mathrm{MHz}$ high-resolution micro-ultrasound in real-time targeting of transrectal prostate biopsies: initial experience. World J Urol. 2020. doi:10.1007/s00345-01902863-y

[14] Saita A, Lughezzani G, Buffi NM, et al. Assessing the Feasibility and Accuracy of High-resolution Microultrasound Imaging for Bladder Cancer Detection and Staging. Eur Urol. 2019:1-6. doi:10.1016/j.eururo.2019.03.044

[15] Bottero $M$, Alì E, Demofonti C, et al. High-resolution microultrasound imaging for bladder cancer: the birth of a new diagnostic tool? Transl Androl Urol. 2019;8(S5):S505S506. doi:10.21037/tau.2019.08.20

[16] Pan D, Soloway MS. The importance of transurethral resection in managing patients with urothelial cancer in the bladder: Proposal for a transurethral resection of bladder tumor checklist. Eur Urol. 2012;61(6):1199-203. doi:10.1016/ j.eururo.2012.03.018

[17] Hurle R, Peschechera R, Buffi NM, et al. Impact of a teaching program on outcome quality of white light transurethral resection for bladder tumor: A cohort study. J Solid Tumors. 2016. doi:10.5430/jst.v6n2p56

[18] Hurle R, Lazzeri M, Colombo P, et al. "En Bloc" Resection of Nonmuscle Invasive Bladder Cancer: A Prospective Single-center Study. Urology. 2016. doi:10.1016/j.urology. 2016.01.004
[19] Moch H, Humphrey PA, Ulbright TM, Reuter VE. WHO classification of tumours of the urinary system and male genital organs. World Heal Organ classifcation tumours. 2016.

[20] Sobin LH, Gospodarowicz MK, Wittekind C. TNM classification of malignant tumors. UICC International Union Against Cancer, ed. 7 BT - TNM classification of malignant tumors. UICC International Union Against Cancer, ed. 7. In: TNM Classification of Malignant Tumors. UICC International Union Against Cancer, Ed. 7.; 2009.

[21] Nicolau C, Bunesch L, Peri L, et al. Accuracy of contrastenhanced ultrasound in the detection of bladder cancer. $\mathrm{Br}$ J Radiol. 2011. doi:10.1259/bjr/43400531

[22] Stamatiou K, Papadoliopoulos I, Dahanis S, Zafiropoulos G, Polizois K. The accuracy of ultrasonography in the diagnosis of superficial bladder tumors in patients presenting with hematuria. Ann Saudi Med. 2009. doi:10.4103/02564947.51802

[23] Drudi FM, Di Leo N, Malpassini F, Antonini F, Corongiu $\mathrm{E}$, Iori $\mathrm{F}$. CEUS in the differentiation between low and high-grade bladder carcinoma. J Ultrasound. 2012. doi: 10.1016/j.jus.2012.09.002

[24] Li Q, Tang J, He E, Li Y, Zhou Y, Wang B. Differentiation between high- and low-grade urothelial carcinomas using contrast enhanced ultrasound. Oncotarget. 2017. doi: 10.18632/oncotarget.20151

[25] Wang HJ, Pui MH, Guan J, et al. Comparison of early submucosal enhancement and tumor stalk in staging bladder urothelial Carcinoma. Am J Roentgenol. 2016. doi:10.2214/ AJR.16.16283

[26] Barchetti G, Simone G, Ceravolo I, et al. Multiparametric MRI of the bladder: inter-observer agreement and accuracy with the Vesical Imaging-Reporting and Data System (VI-RADS) at a single reference center. Eur Radiol. 2019;29(10):5498-506. doi:10.1007/s00330-019-06117-8

[27] Del Giudice F, Barchetti G, De Berardinis E, et al. Prospective Assessment of Vesical Imaging Reporting and Data System (VI-RADS) and Its Clinical Impact on the Management of High-risk Non-muscle-invasive Bladder Cancer Patients Candidate for Repeated Transurethral Resection. Eur Urol. 2020;77(1):101-9. doi:10.1016/j.eururo.2019.09.029

[28] Huang L, Kong Q, Liu Z, Wang J, Kang Z, Zhu Y. The diagnostic value of MR imaging in differentiating t staging of bladder cancer: A meta-analysis. Radiology. 2018. doi:10.1148/radiol.2017171028

[29] Thoeny HC, Bellin MF, Comperat EM, Thalmann GN. Vesical Imaging-Reporting and Data System (VI-RADS): Added Value for Management of Bladder Cancer Patients? Eur Urol. 2018. doi:10.1016/j.eururo.2018.06.017

[30] Ghai S, Eure G, Fradet V, et al. Assessing Cancer Risk on Novel $29 \mathrm{MHz}$ Micro-Ultrasound Images of the Prostate: Creation of the Micro-Ultrasound Protocol for Prostate Risk Identification. J Urol. 2016. doi:10.1016/j.juro.2015.12.093

[31] Mueller-Lisse U, Mueller-Lisse U, Scheidler J, Klein G, Reiser M. Reproducibility of image interpretation in MRI of the prostate: Application of the sextant framework by two different radiologists. Eur Radiol. 2005. doi:10.1007/ s00330-005-2695-z

[32] Nogueira L, Wang L, Fine SW, et al. Focal Treatment or Observation of Prostate Cancer: Pretreatment Accuracy of Transrectal Ultrasound Biopsy and T2-weighted MRI. Urology. 2010. doi:10.1016/j.urology.2009.04.061 